\title{
Coherent Transport in a Periodically Driven Bistable System
}

Frank Grossmann, ${ }^{1,2}$ Thomas Dittrich, ${ }^{1}$ Peter Jung, ${ }^{1}$ and Peter Hänggi ${ }^{1}$

\begin{abstract}
The quantum dynamics of a quartic double well, subjected to a harmonically oscillating field, is studied in the framework of the Floquet formalism. The modifications of the familiar tunneling process due to the driving are investigated numerically and explained in terms of the structure of the corresponding local quasienergy spectrum. In particular, there is a one-dimensional manifold in the parameter space spanned by the amplitude and frequency of the driving force, where tunneling is almost completely suppressed by the coherent driving. The quantal dynamics in the semiclassical regime as well as the influence of weak incoherent processes are briefly discussed.
\end{abstract}

KEY WORDS: Driven tunneling; Floquet theory; localization; exact and avoided crossings; noisy quantum dynamics.

\section{INTRODUCTION}

Bistable systems are abundant in physics, from the microscopic to the macroscopic realm. On the macroscopic level, bistability represents a basic concept in nonlinear dynamics. In quantum mechanics, on the other hand, bistable potentials are associated with a paradigmatic coherence effect: tunneling. ${ }^{(1)}$

Accordingly, this class of systems represents a particularly promising field to study the interplay of classical nonlinearity and quantum coherence and the way it is reflected in phase-space transport.

In the present work we investigate the influence of periodic driving on the quantal dynamics in a bistable potential. Being equivalent to adding one more degree of freedom, external driving is capable of qualitatively altering the dynamics: e.g., in the classical limit, it can render a bistable system chaotic. ${ }^{(2,3)}$

\footnotetext{
${ }^{1}$ Department of Physics, University of Augsburg, W-8900 Augsburg, Germany.

${ }^{2}$ Present address: Department of Chemistry BG-10, University of Washington, Seattle, Washington 98195.
} 
However, only periodic driving is simple enough to still allow, by way of its discrete translation symmetry, for a systematic analytical treatment: The Floquet formalism provides a generalization of the notions of energy eigenvalues and eigenstates to periodically time-dependent systems and thus enables the use of the associated formal tools (e.g., spectral analysis $).{ }^{(4-8)}$

Specific questions we address concern the fate of the ground-state doublet under periodic driving and the concomitant modifications of the familiar tunneling process, as well as, in an advanced stage of the project, the way the system approaches its classical limit, both in the sense of $\hbar$ becoming small compared to characteristic actions and in the sense of incoherent processes entering the stage.

In Section 2, we present our working example, a harmonically driven quartic double well, and introduce some analytical concepts for later reference, such as the Floquet formalism and the local spectrum. Section 3 contains our principal results. They form a survey of the coherence phenomena that replace tunneling in various regimes of the parameter space spanned by the amplitude and frequency of the driving force. A prominent and surprising example, to be discussed in some detail, is the almost complete suppression of tunneling along one-dimensional manifolds in that parameter space. Aspects of the classical limit - the influence of chaotic classical dynamics and of incoherent processes induced by the environment-are briefly addressed in Section 4. In Section 5, we give a summary of our results and add a note on possible experimental realizations.

This contribution is partially based on results originally published in earlier works by the present authors. ${ }^{(9-11)}$

\section{THE PERIODICALLY DRIVEN DOUBLE WELL}

The system we study is a quartic double-well potential driven by a monochromatic force. Its Hamiltonian reads, in dimensionless variables,

$$
\begin{aligned}
H(x, p ; t) & =H_{0}(x, p)+H_{1}(x ; t) \\
H_{0}(x, p) & =\frac{p^{2}}{2}-\frac{x^{2}}{4}+\frac{x^{4}}{64 D} \\
H_{1}(x ; t) & =S x \cos \omega t
\end{aligned}
$$

where $D$ denotes the barrier height, and $S$ and $\omega$ are the amplitude and frequency of the driving force, respectively. 
In order to discuss the symmetries characterizing this system, it is helpful to treat the Hamiltonian (2.1) as if it were an autonomous one. ${ }^{(12,13)}$ This is achieved by giving time the character of an additional spatial degree of freedom, with energy $\hat{E}=-i \partial / \partial t$ its conjugate momentum. Correspondingly, the Hilbert space is extended to the product of the set of square-integrable functions on the strip $-\infty<x<\infty$ and the set of $T$-periodic functions on the interval $0 \leqslant t<T$, where $T=2 \pi / \omega$ is the period of the driving force. The original Hamiltonian (2.1) is replaced by

$$
\mathscr{H}(x, p ; t, E)=H(x, p ; t)+\hat{E}=H(x, p ; t)-i \frac{\partial}{\partial t}
$$

The invariance of the system under discrete translations $t \rightarrow t+n T$, $n=0, \pm 1, \pm 2, \ldots$, left by the periodic driving, can now be exploited in complete analogy to the discrete spatial invariance of crystalline solids, with all its far-reaching consequences.

By the same reasoning that leads to Bloch's theorem ${ }^{(14)}$ in the solidstate context, one derives Floquet's theorem, its analog for periodically time-dependent systems. ${ }^{(15-18)}$ It states that solutions of the Schrödinger equation for the extended Hamiltonian (2.2),

$$
\mathscr{H}\left|\psi_{\alpha}\right\rangle=0
$$

can be written in the form

$$
\psi_{\alpha}(x ; t)=\exp \left(-i \varepsilon_{\alpha} t\right) \phi_{\alpha}(x ; t), \quad \phi_{\alpha}(x ; t+n T)=\phi_{\alpha}(x, t)
$$

The eigenvalues $\varepsilon_{\alpha}$ are called quasienergies. In fact, each of them is a representative of an infinite class of eigenvalues $\varepsilon_{\alpha, k}=\varepsilon_{\alpha}+k \omega, k=0, \pm 1, \pm 2, \ldots$, as is obvious if one expands $\phi_{x}(x, t)=\sum_{k} \phi_{\alpha, k}^{(x)} \exp (-i k \omega t)$. In other words, the quasienergy spectrum is cyclic, i.e., defined $\bmod \omega$, similar to the Brillouin zone structure in the solid-state context.

Again in analogy to the Bloch case, the eigenfunctions (2.4) are simultaneously eigenfunctions of the group of unitary operators associated with the translational symmetry in time, i.e.,

$$
U\left|\psi_{\alpha}\right\rangle=\exp \left(-i \varepsilon_{\alpha} T\right)\left|\psi_{\alpha}\right\rangle
$$

The Floquet operator $U$ is defined as the propagator over one period of the driving force,

$$
U=U(T, 0)=\mathscr{T} \exp \left(-i \int_{0}^{T} d t H(t)\right)
$$


where $\mathscr{T}$ denotes the time-ordering operator. The property

$$
U^{n}\left|\psi_{\alpha}(0)\right\rangle=U(n T, 0)\left|\psi_{\alpha}(0)\right\rangle=\left|\psi_{\alpha}(n T)\right\rangle
$$

shows that $U$ provides a stroboscopic description of the time evolution and this justifies calling $U$ a "quantum map."

Another, more special symmetry of the system described by the Hamiltonian (2.1) goes back to the inversion symmetry $x \rightarrow-x, p \rightarrow-p$, of phase space for the time-independent system $H_{0}(x, p)$. This symmetry is destroyed by an arbitrary driving term, but for the harmonic time dependence chosen here, the relation $\cos (\omega t+\pi)=-\cos (\omega t)$ allows for invariance under the operation

$$
\mathscr{P}: \quad x \rightarrow-x, p \rightarrow-p, t \rightarrow t+\frac{T}{2}
$$

$\mathscr{P}$ forms a unitary symmetry and may be regarded as a parity generalized to the extended Hilbert space introduced above. As a consequence, quasienergy eigenstates are either even or odd under $\mathscr{P}$,

$$
\mathscr{P} \psi_{\alpha}(x ; t)= \pm \psi_{\alpha}(x ; t)
$$

which establishes two symmetry classes of eigenstates. Invariance under $\mathscr{P}$ can be destroyed both by lifting the inversion symmetry of $H_{0}(x, p)$ or by choosing a time dependence $f(t)$ of the driving force such that $f(t+T / 2)=-f(t)$ no longer holds.

A quantity that provides some condensed information on the transport of probability between the two wells of the bistable potential, and that allows one to relate this information directly to the relevant structures in the quasienergy spectrum, is the probability to return, ${ }^{(19,20)}$

$$
P^{\left(\psi_{0}\right)}(t)=\left|\left\langle\psi(t) \mid \psi_{0}\right\rangle\right|^{2}
$$

defined with reference to some initial state $\left|\psi_{0}\right\rangle$. Restricting time to a stroboscopic, discrete series $t_{n}=n T, n=0, \pm 1, \pm 2, \ldots$, and expressing the time evolution by means of the Floquet operator $U$ defined in Eq. (2.6), we can write the probability to return as

$$
P^{\left(\psi_{0}\right)}(n)=\left|\left\langle\psi_{0}\left|U^{n}\right| \psi_{0}\right\rangle\right|^{2}
$$

The role of the quasienergies for this time evolution is made explicit by expanding Eq. (2.11) in the basis of the quasienergy eigenstates,

$$
P^{\left(\psi_{0}\right)}(n)=\xi^{-1}+\sum_{\alpha \neq \beta} \exp \left[i\left(\varepsilon_{\alpha}-\varepsilon_{\beta}\right) n T\right]\left|\left\langle\psi_{\alpha} \mid \psi_{0}\right\rangle\right|^{2}\left|\left\langle\psi_{\beta} \mid \psi_{0}\right\rangle\right|^{2}
$$


Here, $\xi^{-1}$, the diagonal part of the double sum in Eq. (2.12), gives the long-time average of $P^{\left(\psi_{0}\right)}(n)$,

$$
\xi^{-1}=\sum_{\alpha}\left|\left\langle\psi_{\alpha} \mid \psi_{0}\right\rangle\right|^{4}=\lim _{N \rightarrow \infty} \frac{1}{2 N+1} \sum_{n=-N}^{N} P^{\left(\psi_{0}\right)}(n)
$$

It is referred to as the inverse participation ratio. By Fourier transformation, the probability to return is related to the spectral two-point correlation of the local spectrum, ${ }^{(19-22)}$

$$
\begin{aligned}
P_{2}^{\left(\psi_{0}\right)}(\eta)= & \frac{1}{2 \pi} \sum_{n=-\infty}^{\infty} \exp (-i \eta n)\left[P^{\left(\psi_{0}\right)}(n)-\xi^{-1}\right] \\
= & \sum_{\alpha \neq \beta}\left|\left\langle\psi_{\alpha} \mid \psi_{0}\right\rangle\right|^{2}\left|\left\langle\psi_{\beta} \mid \psi_{0}\right\rangle\right|^{2} \\
& \times \int_{0}^{2 \pi} d \Omega \delta\left(\Omega+\frac{\eta}{2}-\varepsilon_{\alpha} T\right) \delta\left(\Omega-\frac{\eta}{2}-\varepsilon_{\beta} T\right)
\end{aligned}
$$

where $\eta,-\pi \leqslant \eta<\pi$, is a scaled quasienergy argument. As is obvious from Eq. $(2.14), P_{2}^{\left(\psi_{0}\right)}(\eta)$ is not an unbiased spectral correlation function, but endows each pair of quasienergies with a weight equal to the product of the respective overlaps of the associated eigenstates with the initial state $\left|\psi_{0}\right\rangle$ chosen. In other words, it weights the components of the spectrum with respect to their relevance for a particular dynamics as it evolves from a specific initial preparation of the system.

\section{DRIVEN TUNNELING}

In the present section, we discuss the modifications imposed on the familiar tunneling dynamics due to periodic driving. That is, we concentrate on the time evolution, under the external force, of a state that is initially prepared as an approximation to a superposition of the two lowest unperturbed eigenstates, $\left|\psi_{L / R}\right\rangle=\left(\left|\psi_{1}\right\rangle \pm\left|\psi_{2}\right\rangle\right) / \sqrt{2}$, centered in one of the two wells. Accordingly, we trace the quasienergy doublet that corresponds to the unperturbed energies $E_{1}$ and $E_{2}$ through the parameter space spanned by amplitude $S$ and frequency $\omega$ of the driving force. Thereby, we disregard effects due to a preparation of the initial state that includes significant contributions from higher-lying eigenstates. Despite the elementary nature of the dynamics thus specified, we encounter a surprisingly rich repertoire of coherent variations of tunneling.

There are two regimes in the $(S, \omega)$ plane where tunneling is not qualitatively altered: Both in the limits of slow (adiabatic) and of fast driving, the separation of the time scales of inherent dynamics and external 

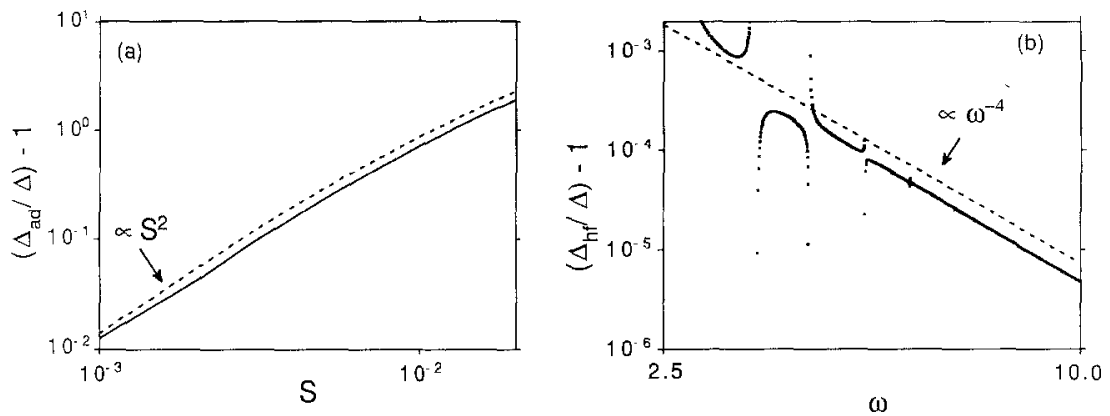

Fig. 1. Driven tunneling in the adiabatic/fast limits. (a) Log-log plot of the deviation of the tunnel splitting from its unperturbed value, for adiabatic driving, as a function of the driving amplitude, (b) log-log plot of the same quantity, for fast driving, as a function of the driving frequency.

force effectively uncouples these two processes and leads to a mere renormalization of the tunneling rate $A(S, \omega)$, given as the splitting of the two quasienergies involved. Specifically, as both an analytical treatment and numerical experiments show, ${ }^{(23)}$ the driving always reduces the effective barrier height and thus augments the tunneling rate in the two limits at issue (Fig. 1).

Qualitative changes in the tunneling behavior are expected as soon as the driving frequency significantly interferes with the internal frequencies of the double well, i.e., in particular, the tunnel splitting $A$ and the so-called resonances $E_{3}-E_{2}, E_{4}-E_{1}, E_{5}-E_{2}, \ldots$ (see Fig. 2a). A more detailed account of this intuitive picture is obtained by constructing the intricate three-dimensional "landscape" of quasienergy planes $\varepsilon_{\alpha, k}(S, \omega)$ :
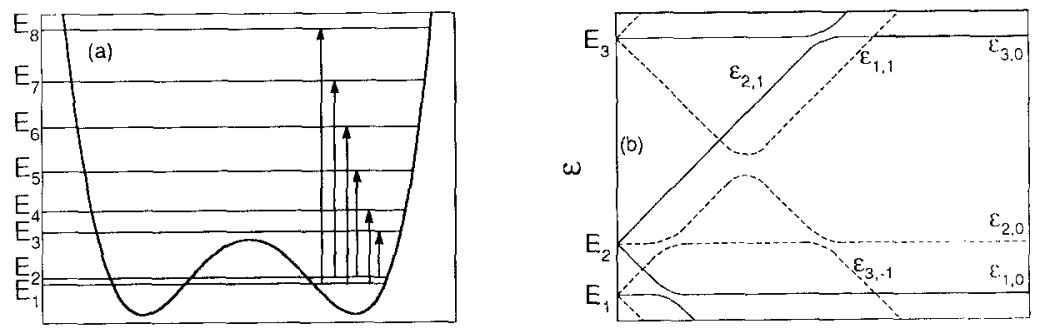

(1)

Fig. 2. Energy and quasienergy levels in the quartic double-well potential. (a) Timeindependent potential with energy eigenvalues, for $D=1$ [arrows indicate transitions from the ground-state doublet to excited states, allowed by parity conservation (resonances)]; (b) schematic plot of relevant quasienergies, as functions of $\omega$, at a small value of $S$ (quasienergies associated with eigenstates of even/odd parity are indicated by full/dashed lines; energy differences are not to scale to make the crossing structure visible). 
As a starting point, consider the case $S=0$, where the driving is switched off. Here, the quasienergies are exactly given by the linear dependence

$$
\varepsilon_{\alpha, k}=E_{\alpha}+k \omega, \quad k=0, \pm 1, \pm 2, \ldots
$$

As pointed out in the previous section, this infinite multiplicity is a consequence of the fact that there is an infinity of equivalent ways to construct the Floquet solutions in the form given by Eq. (2.4); it is lifted if the quasienergies are considered mod $\omega$. Physical significance can only be ascribed to the difference $\Delta k=k^{\prime}-k$ of the $k$-indices of a pair of quasienergies $\varepsilon_{\alpha, k}, \varepsilon_{\alpha^{\prime}, k^{\prime}}$. In particular, crossings $\varepsilon_{\alpha, k} \approx \varepsilon_{\alpha^{\prime}, k^{\prime}-\Delta k}$ can be considered as $\Delta k$-photon transitions between levels of the undriven system if a quantumoptical interpretation of the periodic driving is adopted. This makes it immediately clear that for $S>0$, where Eq. (3.1) is no longer exactly valid, it is the quasienergy crossings where the driving is felt most strongly. Actually, this statement has to be qualified by two restrictions:

First, by an argument going back to von Neumann and Wigner, ${ }^{(24,25)}$ two parameters must be varied independently to locate an accidental energy degeneracy in a time-reversal-invariant system. This implies that quasienergy crossings are found at isolated points in the $(S, \omega)$ plane, and a one-dimensional section through this plane will typically show avoided crossings. This is true, however, only within each of the two parity classes of eigenstates mentioned in the previous section [see Eq. (2.9)]; crossings between quasienergies associated with eigenstates of opposite parity may form one-dimensional manifolds in the $(S, \omega)$ plane and thus show up as exact crossings in sections through the $\varepsilon(S, \omega)$ space along arbitrary lines in the $(S, \omega)$ plane.

Second, the effective coupling, due to the driving, between two unperturbed levels at a crossing $E_{\alpha}=E_{\alpha^{\prime}}-\Delta k \omega$, as reflected in the degree of splitting of that crossing for finite $S$, rapidly decreases with increasing $\Delta k$, namely as $S^{\Delta k}$. This is suggested by the interpretation as a $\Delta k$-photon transition and can be substantiated using $\Delta k$ th-order perturbation theory. ${ }^{(26)}$ Consequently, in the regime of small $S$, only crossings with $\Delta k$ a small number exhibit significant splittings.

The few rules stated in the previous paragraphs are sufficient to provide a semiquantitative understanding of the structure of the quasienergy spectrum as a function of $\omega$, at least for small $S$, as it is revealed by numerical diagonalization ${ }^{3}$ of the Floquet operator $U$ (the validity of our

\footnotetext{
${ }^{3}$ For the numerical calculation of the quasienergy spectrum we adopted the method of matrix continued fractions described in ref. 27.
} 
numerical treatment is, of course, not restricted to small $S$ ). A schematic representation of this structure is given in Fig. 2b.

As an application, we discuss two specific "samples" of the quasienergy spectrum with the corresponding tunneling dynamics, one of them featuring an avoided crossing, the other an exact one.

The "single-photon transition" at $\omega=E_{3}-E_{2}$ is called the fundamental resonance. It corresponds to a crossing between the quasienergies $\varepsilon_{2, k}$ and $\varepsilon_{3, k-1}$ and, for $S>0$, forms an avoided crossing, since the corresponding eigenstates have equal parity. Figure $3 a$ shows the time evolution of the probability to return, $P^{\left(\psi_{0}\right)}(n)$ [see Eq. (2.11)], at the fundamental resonance (with $S=10^{-4}$ ), for an initial state prepared as the ground state of a harmonic oscillator approximating one of the wells, i.e., a Gaussian approximation of $\left|\psi_{L / R}\right\rangle$. The monochromatic oscillation of $P^{\left(\psi_{0}\right)}(n)$ characteristic of unperturbed tunneling has given way to a more complex beat pattern. Its Fourier transform, the two-point correlation $P_{2}^{\left(\psi_{0}\right)}(\eta)$ of the local spectrum [see Eq. (2.14)] reveals that this beat pattern is mainly composed of two groups of three frequencies each, which in turn can be identified as the quasienergy differences $\varepsilon_{3,-1}-\varepsilon_{2,0}, \varepsilon_{2,0}-\varepsilon_{1,0}, \varepsilon_{3,-1}-\varepsilon_{1,0}$ (Fig. $3 b$ ), and $\varepsilon_{4,-1}-\varepsilon_{3,-1}, \varepsilon_{4,-1}-\varepsilon_{2,0}, \varepsilon_{4,-1}-\varepsilon_{1,0}$, at the avoided crossing (Figs. $3 \mathrm{c}$ and $3 \mathrm{~d}$ ).
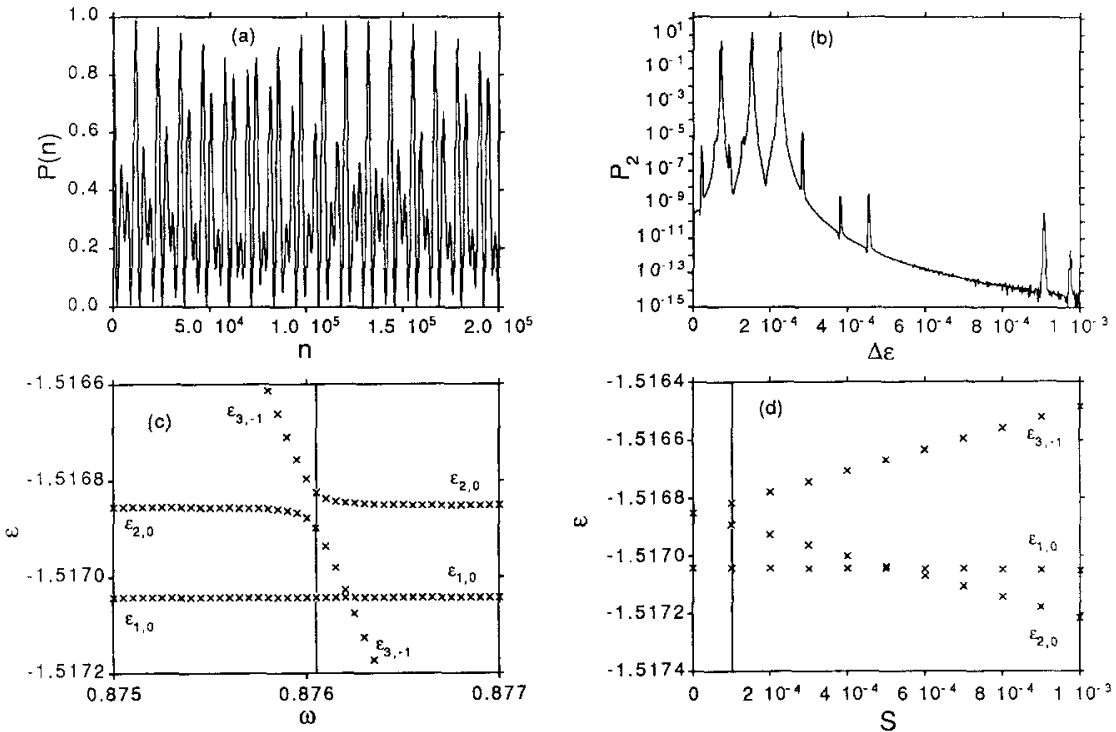

Fig. 3. Driven tunneling near the fundamental resonance, $\omega=E_{3}-E_{2}$, at $S=10^{-4}$. (a) Time evolution of $P^{\left(\psi_{0}\right)}(n)$ over the first $2 \times 10^{5}$ time steps; (b) local spectral two-point correlation as obtained from (a) (only the lower one of two triplets is shown); (c) quasienergy spectrum, as a function of $\omega$ near $\omega=E_{3}-E_{2}$, at $S=10^{-4}$; (d) quasienergy spectrum, as a function of $S$ near $S=10^{-4}$, at $\omega=E_{3}-E_{2}$. 
In contrast, a two-photon transition that bridges the tunnel splitting $\Delta=E_{2}-E_{1}$ is "parity forbidden," and thus the quasienergies $\varepsilon_{1, k+1}$ and $\varepsilon_{2, k-1}$ give rise to an exact crossing. Equation (2.12) indicates that a vanishing of the difference $\varepsilon_{2,-1}-\varepsilon_{1,1}$ will have a drastic consequence: For a state prepared as an exact superposition of the corresponding two quasienergy eigenstates only, $P^{\left(\psi_{0}\right)}(t)$ and all other observables become constants, at least at discrete times $n T$, and thus it is possible that tunneling comes to a standstill! As discussed above, exact crossings should occur along one-dimensional manifolds in the $(S, \omega)$ plane. Figure 5 shows such a manifold for $\varepsilon_{2,-1}=\varepsilon_{1,1}$, as determined numerically: It is a closed curve, reflection-symmetric with respect to the line $S=0$, with an approximately linear frequency dependence for $\Delta \lesssim \omega \lesssim E_{3}-E_{2}$. Furthermore, simulations of the dynamics show that along the linear part of that manifold, the second necessary condition for a complete suppression of tunneling-that the inherent time dependence of the quasienergy eigenstates, with period $T$, vanish as well-is indeed approximately fulfilled. For $\Delta / 2 \lesssim \omega \lesssim \Delta$, on the other hand, $S$ becomes so small on the manifold that the dynamics approaches that of the undriven case, while for $\omega \rightarrow E_{3}-E_{2}$, the strong participation of a third quasienergy introduces nonzero frequencies into the time dependence.

Various aspects of the suppression of tunneling are elucidated in Fig. 4. A typical time evolution of $P^{\left(\psi_{0}\right)}(n)$ demonstrating the suppression of tunneling is presented in Fig. 4a. In fact, oscillations of small amplitude remain; they can be ascribed to an admixture of higher-lying quasienergy states to the initial state, as the local spectrum (Fig. 4c) shows. In addition, the time dependence synchronous with the driving frequency has not completely vanished, as is revealed by the resolved evolution of $P^{\left(\psi_{0}\right)}(t)$ within a single period of the driving force (Fig. 4b). Finally, a space-resolved comparison of $\psi(x, t)$ with the initial state, at a time $(n=458)$ where the deviation of $P^{\left(\psi_{0}\right)}(t)$ from unity is exceptionally large (Fig. 4d), confirms that the leakage of probability into the initially empty, opposite well indeed remains extremely small: So the coherent suppression of tunneling truly amounts to a localization of the wave packet in one of the wells.

Surprising as it is, this phenomenon appears to be an elementary quantum interference effect. As a matter of fact, much of it can be understood on basis of a two-state approximation

$$
H(t)=\frac{A}{2}\left(-\left|\psi_{1}\right\rangle\left\langle\psi_{1}|+| \psi_{2}\right\rangle\left\langle\psi_{2}\right|\right)+A \cos \omega t\left(\left|\psi_{1}\right\rangle\left\langle\psi_{2}|+| \psi_{2}\right\rangle\left\langle\psi_{1}\right|\right)
$$

where $\Delta$ is again the tunnel splitting and $A=S\left\langle\psi_{1}|x| \psi_{2}\right\rangle$ is the effective strength of the perturbation. In the present case, it is more appropriate to 

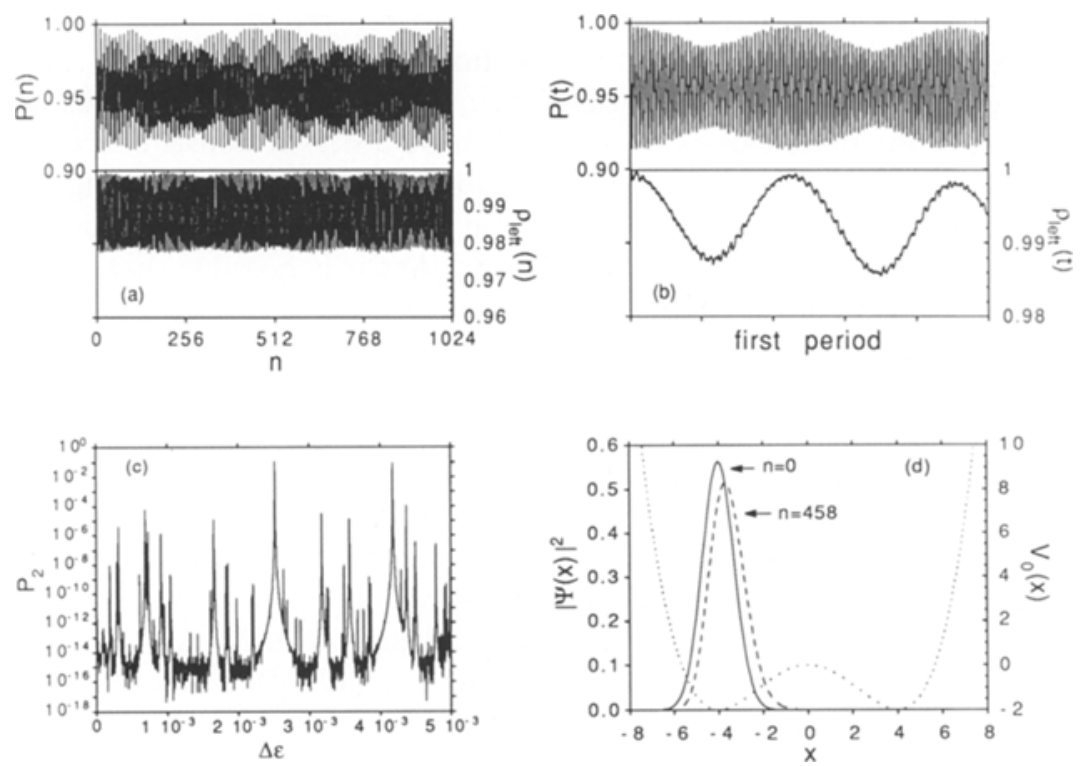

Fig. 4. Suppression of tunneling due to a crossing of $\varepsilon_{1,1}$ and $\varepsilon_{2,-1}$, at $\omega=0.01$ and $S=3.171 \times 10^{-3}$. (a) Time evolution of $P^{\left(\psi_{0}\right)}(n)$ over the first $2^{10}$ time steps; (b) resolved time evolution of $P^{\left(\psi_{0}\right)}(t)$ over a single period of the driving force; (c) local spectral two-point correlation function $P_{2}^{\left(\psi_{0}\right)}(n)$ as obtained from (a); (d) space-resolved state at $n=458$ (dashed line), compared with the initial state (full line), the dotted line indicating the unperturbed potential.

use the basis provided by the states $\left|\psi_{L / R}\right\rangle=\left(\left|\psi_{1}\right\rangle \pm\left|\psi_{2}\right\rangle\right) / \sqrt{2}$. The Hamiltonian then takes the form

$$
H(t)=\frac{A}{2}\left(\left|\psi_{L}\right\rangle\left\langle\psi_{R}|+| \psi_{R}\right\rangle\left\langle\psi_{L}\right|\right)+A \cos \omega t\left(\left|\psi_{L}\right\rangle\left\langle\psi_{L}|-| \psi_{R}\right\rangle\left\langle\psi_{R}\right|\right)
$$

so that the equations of motion for the expansion coefficients of an arbitrary state $|\psi(t)\rangle=c_{L}(t)\left|\psi_{L}\right\rangle+c_{R}(t)\left|\psi_{R}\right\rangle$ are

$$
\begin{aligned}
& \dot{c}_{L}(t)=-i\left[A \cos \omega t c_{L}(t)+\frac{A}{2} c_{R}(t)\right] \\
& \dot{c}_{R}(t)=-i\left[-A \cos \omega t c_{R}(t)+\frac{A}{2} c_{L}(t)\right]
\end{aligned}
$$

An approximate solution of this pair of coupled differential equations is obtained if incomplete localization, i.e., $0 \leqslant c_{R}(t) \ll c_{L}(t) \leqslant 1$, for all times $t$, 
is assumed as an ansatz. The contribution of $c_{R}(t)$ to the equation of motion of $c_{L}(t)$ can then be neglected, allowing for an analytic solution of Eq. (3.4). It reads, for the right-hand well coefficient at stroboscopic times $n T$,

$$
c_{R}(n T)=-i \frac{\Delta}{2} J_{0}\left(\frac{2 A}{\omega}\right)
$$

where $J_{0}(x)$ denotes the ordinary Bessel function of zeroth order. Equation (3.5) immediately implies that a suppression of tunneling is to be expected if the condition

$$
S(\omega)=\frac{\omega}{2\left\langle\psi_{1}|x| \psi_{2}\right\rangle} a_{j}
$$

is fulfilled, where $a_{j}$ is the $j$ th zero of $J_{0}(x)$.

A comparison of the function $S(\omega)$ given by Eq. (3.6) with the numerically determined manifold for localization to occur (Fig. 5) shows that this function, for $j=1$, reproduces the linear section of that manifold. Moreover, analogous manifolds, given by the subsequent zeros $a_{j}$, where exact crossings of $\varepsilon_{2,-j}$ with $\varepsilon_{1, j}, j=2,3, \ldots$, occur, are indeed found where predicted by Eq. (3.6). ${ }^{(28)}$ Finally, the decay $\sim x^{-1 / 2}$, for large arguments, of the modulus of $J_{0}(x)$ implies that within the two-state approximation, tunneling should always be suppressed for sufficiently large $S$.

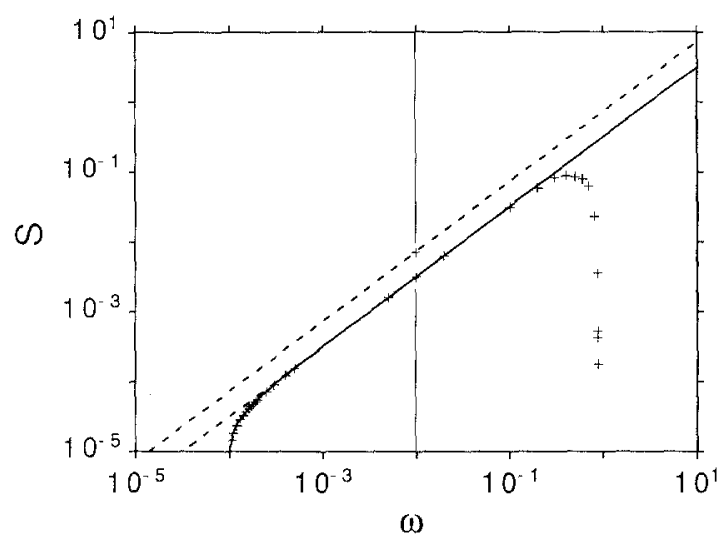

Fig. 5. Two of the manifolds in the $(S, \omega)$ plane where a suppression of tunneling occurs. Data obtained by diagonalization of the full Floquet operator for the driven double well are indicated by crosses, the full line is the result of an exact solution of the corresponding twostate model (3.3), and the dashed lines have been obtained by the approximate condition (3.6) derived from the two-state model (3.3). 
The two-state approximation does not account, however, for the section of the localization manifold that bends back to $S=0$ for $\omega \approx E_{3}-E_{2}$, due to the approach of a third quasienergy, and of course, it cannot provide any detailed information as to the spatial aspects of tunneling.

\section{ASPECTS OF THE CLASSICAL LIMIT}

While the previous section has dealt with driven tunneling in the deep quantum regime, we shall now discuss issues that are associated with the approach toward the macroscopic realm. This includes at least two different aspects: the increase in the phase-space scales characterizing the system that allows the use of small-wavelength approximations and lets finer and finer details of the classical phase space influence the quantum dynamics, as well as the growing role of ambient degrees of freedom that tend to reduce the complexity of the dynamics by destroying the subtler quantum coherence effects.

A quantitative measure for the position of the system between the quantal and the classical limit is given by the ratio of some action that characterizes the scales of the classical structures in phase space and Planck's constant $\hbar$. Writing the unperturbed Hamiltonian of the quartic double well in an unscaled form,

$$
H_{0}(x, p)=\frac{p^{2}}{2 m}-\frac{m \omega_{0}^{2}}{4} x^{2}+\frac{m^{2} \omega_{0}^{2}}{64 E_{B}} x^{4}
$$

where $\omega_{0}$ is the frequency of oscillations on the bottom of each of the wells and $E_{B}$ is the barrier height, we can extract a characteristic action as

$$
S=\frac{E_{B}}{\omega_{0}}
$$

It is proportional to the volume of phase space encircled by the separatrix that separates motion within the wells from motion above the barrier. Consequently, the classical limit is approached if

$$
\hbar_{\mathrm{rel}}=\frac{\hbar}{S}=\frac{\hbar \omega_{0}}{E_{B}}
$$

becomes small. According to the scaling used to obtain the Hamiltonian (2.1) from the version (4.1), the identity $\hbar_{\text {rel }}=1 / D$ holds, so that, with the parameters used in Sections 2 and $3, D \rightarrow \infty$ corresponds to the classical limit. This is plausible since $D$ approximately gives the number of unperturbed eigenenergy pairs below the barrier. 
As soon as the driving is switched on, the classical motion becomes chaotic. In accordance with the KAM theorem, integrability is destroyed first, i.e., for small $S$, in the vicinity of the separatrix. ${ }^{(2,3)}$ A further increase of $S$ lets the integrable areas around the potential minima shrink until they disappear completely. Within the chaotic "sea," classical motion is diffusive. In this way, a classical transport mechanism between the wells arises that competes with tunneling ${ }^{(29)}$ : A central question, therefore, is how these two mechanisms will interact with one another.

Figure 6 gives an impression of the changes driven tunneling under-
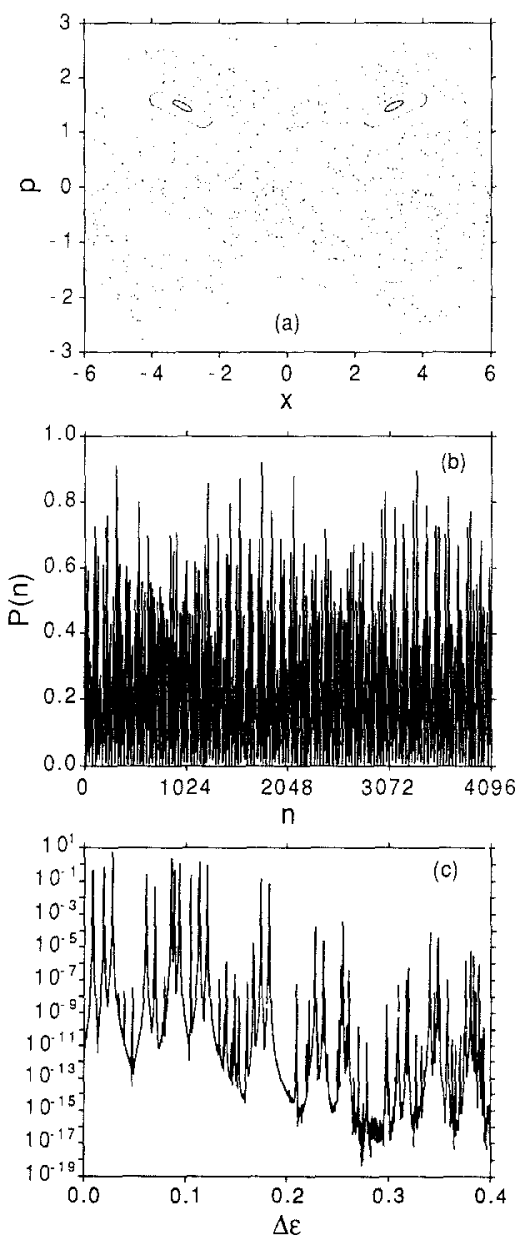

Fig. 6. Driven tunneling at parameter values where the corresponding classical dynamics is dominated by chaos. (a) Classical phase-space portrait, at discrete times $t_{n}=n T$; (b) time evolution of $P^{\left(\psi_{0}\right)}(n)$ over the first $2^{12}$ time steps; (c) local spectral two-point correlation as obtained from (b). The parameter values are $D=2, S=0.15, \omega=0.876$. 
goes in the classically strongly chaotic regime. As a phase-space portrait [a plot of the classical trajectories $(x(t), p(t))$ at discrete times $n T$; Fig. 6a] shows, the integrable islands on the bottom of each well have almost disappeared at the parameter values chosen $(D=2, S=0.15, \omega=0.876)$.

The time evolution of the probability to return (Fig. 6b) looks much more irregular, compared, e.g., to the beat pattern shown in Fig. 3a, where only $S$ differs from the present value $\left(S=10^{-4}\right.$ in Fig. 3). This is consistent with the structure of the local spectrum (Fig. 6c). It shows a large number of significant peaks, whereas the beat pattern of Fig. 3a is dominated by just six frequencies. However, with $D=2$, the case shown here is still in the quantal regime, where specific features of the dynamics cannot yet be unambiguously related to structures in classical phase space.

From the various methods available to incorporate the influence of the ambient degrees of freedom into a quantal dynamics, we restrict ourselves here to the crudest one: Adding a noisy component to the unitary time evolution of the system. ${ }^{(30-33)}$ This can be realized, e.g., by contaminating the deterministic periodic driving $f(t)=S \cos (\omega t)$ by noise,

$$
f_{\xi}(t)=S \cos (\omega t)+\sigma \sum_{n} \xi_{n} \delta(t-n T)
$$

For simplicity, we have restricted the noise to a series of $\delta$-like kicks, synchronous with the driving frequency, so that $\xi_{n}$ denotes a discrete random process. Again for simplicity, we define it to have unit variance and to be uncorrelated for different times. The noise strength is given by $\sigma$. Clearly, a simple construction like this may serve to study the destruction of coherence by the environment in an unspecific way; it does not allow us to model dissipation nor any microscopic details of the environment. In Fig. 7, we show the results of a few numerical experiments using the noisy driving of Eq. (4.4) in conjunction with the two-state approximation (3.3), i.e.,

$H(t)=\frac{\Delta}{2}\left(\left|\psi_{L}\right\rangle\left\langle\psi_{R}|+| \psi_{R}\right\rangle\left\langle\psi_{L}\right|\right)+F_{\xi}(t)\left(\left|\psi_{L}\right\rangle\left\langle\psi_{L}|+| \psi_{R}\right\rangle\left\langle\psi_{R}\right|\right)$

with $F_{\xi}(t)=\left\langle\psi_{1}|x| \psi_{2}\right\rangle f_{\xi}(t)$. Figure $7 \mathrm{a}$ is based on the unperturbed double well (i.e., $S=0$ ); it demonstrates how the oscillatory flow of probability that characterizes tunneling is damped out on a time scale increasing with decreasing noise strength and gives way to a thermalized state where probability is fluctuating around an equidistribution between the wells.

Figure $7 \mathrm{~b}$ refers to a situation where tunneling is almost, but not completely, suppressed by the coherent driving. Due to a small offset of 

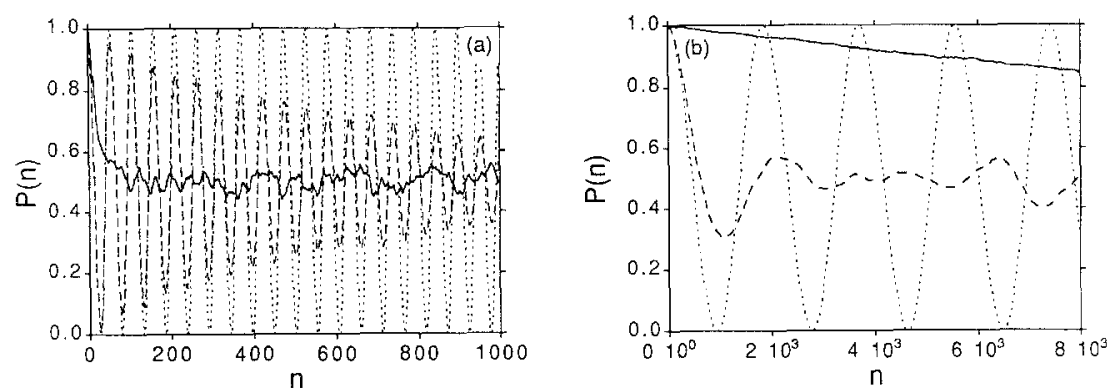

Fig. 7. Influence of noise on driven tunneling. (a) Unperturbed tunneling, without noise (dotted line), with noise of strength $\sigma=0.01$ (dashed line), and with $\sigma=0.1$ (full line); (b) driven tunneling near an exact quasienergy crossing where tunneling is suppressed, same key as for (a). A two-state model [Eq. (3.3)] has been used with parameters $\Delta=1.895 \times 10^{-4}$, and, for (b), $S=3.1 \times 10^{-3}, \omega=0.01$.

the parameters chosen from the manifold where localization occurs, the coherent dynamics consists in tunneling on an extremely long time scale. For weak noise, a damping of the coherent oscillation is again observed. However, for stronger noise, the time scale for a thermalization of probability increases! This means that localization is stabilized by noise, so that the parameter mismatch deliberately chosen appears to be compensated. In fact, with the parameter point placed exactly on the localization manifold, even the slight oscillation of $P^{\left(\psi_{0}\right)}(n)$ that is left in the deterministic case (see Fig. 4a) is suppressed by the noise (no figure).

This surprising behavior resembles the stabilization of classical unstable equilibrium states by multiplicative noise, but it is not quantitatively understood.

\section{SUMMARY}

The present work is intended to give an overview of various aspects of tunneling in a double well under the influence of periodic driving. The basic notions to discuss a periodically driven quantal dynamics are provided by Floquet theory, a time-domain analog of Bloch theory: quasienergies and quasienergy eigenstates replace the familiar concepts of energy eigenvalues and eigenstates, respectively. Consequently, driven tunneling is adequately analyzed in terms of the quasienergies that contribute to the time evolution of a state initially localized in one of the wells.

In the limits of slow and of fast driving, the familiar tunneling dynamics is merely accelerated. Qualitative modifications occur where the quasienergies corresponding to the ground-state doublet of the unperturbed 
double well interact, in parameter space, with additional quasienergies. In particular, avoided crossings can lead to quite complex quantum beat patterns that enhance tunneling, while at specific exact crossings, which form one-dimensional manifolds in parameter space, an almost complete suppression of tunneling occurs. It is essentially a two-quasienergy interference phenomenon; in fact much of it can be understood in terms of a two-state approximation of the double well.

Toward the classical limit, both diffusive transport due to classical chaos and incoherent processes induced by the environment become significant ingredients of the physics of the driven double well. A noisy driving, introduced to crudely account for the influence by the environment, destroys coherent tunneling and leads to a thermalization of probability in the double well. Preliminary results indicate, on the other hand, that coherent localization is stabilized by noise.

Experimental realizations of the driven double well are conceivable in various fields. The ammonia molecule, as the paradigmatic example for tunneling, is well described by a quartic double well with a scaled barrier height $D \approx 2$. For example, in order to see coherent suppression of localization in ammonia, a maser irradiation of wavelength $\lambda \approx 1 \mathrm{~mm}$ and intensity $P \approx 10^{12} \mathrm{~W} / \mathrm{m}^{2}$ would be necessary. A solid-state realization of the driven double well is provided by rf-driven SQUIDs, with an external flux $\phi_{\mathrm{ex}}=\phi_{0} / 2, \phi_{0}=h / 2 e$. For a SQUID with capacitance $C=10^{-15} \mathrm{~F}$, selfinductance $L=10^{-9} \mathrm{H}$, critical current $I_{\mathrm{C}}=10^{-6} \mathrm{~A}$, and corresponding plasma frequency $\omega_{p}=10^{12} \mathrm{~Hz}$, an injected current of frequency $\omega=10^{10} \mathrm{~Hz}$ and amplitude $I=10^{-10} \mathrm{~A}$ should lead to a suppression of tunneling. Alternatively, an oscillating component can be added to the stationary external flux, with the same frequency and an amplitude $\Delta \phi_{\mathrm{ex}}=10^{-19} \mathrm{~Wb}$. Furthermore, any realization of a two-state system with an off-diagonal driving term will serve the same purpose [see Eq. (3.2)]. These realizations naturally involve an interplay of all of the three aspects of transport in a driven bistable system: coherent tunneling, classical chaos, and incoherent processes. Its theoretical understanding is far from complete.

\section{ACKNOWLEDGMENTS}

We acknowledge financial support by the Deutsche Forschungsgemeinschaft through Grant Ha 1517/3-2. One of us (T. D.) would like to thank D. Cohen for a fruitful discussion on noisy quantum dynamics. 


\section{REFERENCES}

1. F. Hund, Z. Phys. 43:803 (1927).

2. A. J. Lichtenberg and M. A. Lieberman, Regular and Stochastic Motion (Springer, New York, 1981).

3. L. E. Reichl and W. M. Zheng, in Directions in Chaos, Vol. 1, Hao Bai-lin, ed. (World Scientific, Singapore, 1987).

4. R. Blümel and U. Smilansky, Phys. Rev. A 30:1040 (1984).

5. N. L. Manakov, V. D. Ovsiannikov, and L. P. Rapoport, Phys. Rep. 141:319 (1986).

6. S. Chu, Adv. Chem. Phys. 73:739 (1986).

7. H. P. Breuer and M. Holthaus, Z. Phys. D 11:1 (1989); Phys. Lett. A 140:507 (1989).

8. M. H. Devoret, D. Esteve, J. M. Martinis, A. Cleland, and J. Clarke, Phys. Rev. B 36:58 (1987).

9. F. Grossmann, P. Jung, T. Dittrich, and P. Hänggi, Z. Phys. B 84:315 (1991).

10. F. Grossmann, T. Dittrich, P. Jung, and P. Hänggi, Phys. Rev. Lett. 67:516 (1991).

11. F. Grossmann, T. Dittrich, and P. Hänggi, Physica B 175:293 (1991).

12. J. Howland, Math. Ann. 207:315 (1974); Lecture Notes in Physics, Vol. 130 (Springer, Berlin, 1980).

13. H. Sambe, Phys. Rev. A 7:2203 (1973).

14. N. W. Ashcroft and N. D. Mermin, Solid State Physics (Holt-Saunders, Tokyo, 1976).

15. J. H. Shirley, Phys. Rev. 138B:979 (1965).

16. Ya. B. Zel'dovich, Zh. Eksp. Teor. Fiz. 51:1492 (1966) [Sov. Phys. JETP 24:1006 (1967)].

17. V. I. Ritus, Zh. Eksp. Teor. Fiz. 51:1544 (1966) [Sov. Phys. JETP 24:1041 (1967)].

18. G. Casati and L. Molinari, Prog. Theor. Phys. Suppl. 98:286 (1989).

19. E. J. Heller, Phys. Rev. A 35:1360 (1987).

20. T. Dittrich and U. Smilansky, Nonlinearity 4:59 (1991).

21. P. W. Anderson, Phys. Rev. 109:1492 (1958); Rev. Mod. Phys. 50:191 (1978).

22. D. R. Grempel, R. E. Prange, and S. Fishman, Phys. Rev. A 29:1639 (1984).

23. F. Grossmann and P. Hänggi, Verh. DPG (VI) 25:691 (1991).

24. J. von Neumann and E. Wigner, Phys. Z. 30:467 (1929).

25. M. V. Berry, in Chaotic Behavior in Quantum Systems: Theory and Applications, G. Casati, ed. (Plenum Press, New York, 1985).

26. A. Gold and H. B. Bebb, Phys. Rev. Lett. 14:60 (1965).

27. H. Risken, The Fokker-Planck Equation (Springer, Berlin, 1984); P. Jung, Z. Phys. B 76:521 (1989).

28. F. Grossmann and P. Hänggi, Europhys. Lett. 18:571 (1992); J. M. Gomez Llorente and J. Plata, Phys. Rev. A 45:R6958 (1992); M. Andrews, P. M. Alsing, and C. M. Savage, preprint (1992).

29. W. A. Lin and L. E. Ballentine, Phys. Rev. Lett. 65:2927 (1990).

30. E. Ott, T. M. Anderson, Jr., and J. D. Hanson, Phys. Rev. Lett. 53:2187 (1984).

31. S. Adachi, M. Toda, and K. Ikeda, Phys. Rev. Lett. 61:655 (1988).

32. B. Meerson, Phys. Rev. Lett. 62:1615 (1989).

33. D. Cohen, Phys. Rev. A 44:2292 (1992). 